\title{
An overview of existing clinical severity scales and genotype-phenotype studies in FSHD
}

\author{
N.V. Zernov ${ }^{1 *}$, M.Yu. Skoblov ${ }^{1,2,3}$ \\ ${ }^{1}$ Research Centre for Medical Genetics, Moscow, Russia \\ ${ }^{2}$ Moscow Institute of Physics and Technology, Dolgoprudny, Russia \\ ${ }^{3}$ Far Eastern Federal University, Vladivostok, Russia \\ *e-mail:nzernov01@gnmail.com
}

Key words: FSHD, genotype-phenotype correlation, clinical severity score, D4Z4 array size, D4Z4 array methylation, disease prognosis, family counselling

Motivation and Aim: Facial-scapular-humeral myodystrophy (FSHD) is an autosomal dominant myodystrophy, the basis of its pathogenesis is ectopic expression of the transcription factor DUX4 in skeletal muscle. DUX4 is encoded by each D4Z4 repeat unit on 4 th chromosome [1]. The prevalence of the disease in different populations varies from 1: 20000 to 1: 14,000 [2]. In this paper, we reviewed existing studies about impact of genetic, epigenetic and gender differences on clinical severity and the possibility of their use for disease prognosis and family counselling.

Methods: To date, there are no specific, fully validated scores for assessing the clinical severity of the FSHD. The most frequently used clinical severity scale was published by Ricci et al. [3] and corrected to age of onset by van Overveld et al. [4].

Results: In most studies of the influence of genetic factors on phenotype, the inverse correlation between the number of the D4Z4 repeats on the 4th chromosome and the severity of clinical manifestations was established [3]. For both types of FSHD, the inverse dependence of the severity of clinical manifestations on the level of methylation of the D4Z4 repeats on 4qA chromosome was shown [5].

Conclusion: Reviewed works allow concluding that the penetrance and the severity of FSHD are in inverse correlation with the number of D4Z4 repeats on the 4qA haplotype and the level of methylation. Moreover, the smaller the residual number of the D4Z4 repeats, the smaller the variability of the clinical picture. In groups of asymptomatic carriers and patients with minimal clinical manifestations are dominated by females. Most researchers conclude that the most accurate prediction could be given with the following data: age of onset, gender, family examination data; clinical severity scoring; the length of D4Z4 repeats on the 4qA chromosome in FSHD1, additionally for FSHD2 presence and type of mutations in the gene SMCHD1; haplotype and methylation status of the array of D4Z4 repeats of chromosome 4.

\section{References}

1. Gabriels J. et al. (1999) Nucleotide sequence of the partially deleted D4Z4 locus in a patient with FSHD identifies a putative gene within each $3.3 \mathrm{~kb}$ element. Gene. 236(1):25-32.

2. Lemmers R.J. et al. (2010) Worldwide population analysis of the $4 q$ and $10 q$ subtelomeres identifies only four discrete interchromosomal sequence transfers in human evolution. Am J Hum Genet. 86(3):364-77.

3. Ricci E. et al. (1999) Progress in the molecular diagnosis of facioscapulohumeral muscular dystrophy and correlation between the number of KpnI repeats at the 4q35 locus and clinical phenotype. Ann Neurol. 45(6):751-7.

4. van Overveld P.G. et al. (2005) Variable hypomethylation of D4Z4 in facioscapulohumeral muscular dystrophy. Ann Neurol. 58(4):569-76.

5. Lemmers R.J. et al. (2015) Inter-individual differences in CpG methylation at D4Z4 correlate with clinical variability in FSHD1 and FSHD2. Hum Mol Genet. 24(3):659-69. 